\title{
Prognostic significance of TERT promoter and BRAF mutations in TIR-4 and TIR-5 thyroid cytology
}

\section{Simona Censi' , Susi Barollo1, Elisabetta Grespan', Sara Watutantrige-Fernando1, Jacopo Manso', Maurizio lacobone ${ }^{2}$, Eric Casal Ide², Francesca Galuppini³, Ambrogio Fassina3, Loris Bertazza', Federica Vianello4, Gianmaria Pennelli³ and Caterina Mian'}

${ }^{1}$ Department of Medicine (DIMED), Endocrinology Unit, University of Padua, Padua, Italy, ${ }^{2}$ Department of Surgical, Oncological and Gastroenterological Sciences (DiSCOG), Endocrine Surgery Unit, University of Padua, Padua, Italy, ${ }^{3}$ Department of Medicine (DIMED), Surgical Pathology and Cytopathology Unit, Pathology Unit, University of Padua, Padua, Italy, and ${ }^{4}$ Department of Radiotherapy, Istituto Oncologico Veneto-IRCCS, Padua, Italy

\section{Correspondence} should be addressed to C Mian

Email

caterina.mian@unipd.it

\begin{abstract}
Objective: Follicular-derived thyroid cancers generally have a good prognosis, but in a minority of cases, they have an aggressive behavior and develop distant metastases, with an increase in the associated mortality. None of the prognostic markers currently available prior to surgery can identify such cases.

Methods: TERT promoter and BRAF gene mutations were examined in a series of 436 consecutive TIR-4 and TIR-5 nodes referred for surgery. Follow-up (median: 59 months, range: 7-293 months) was available for $384 / 423$ patients with malignant nodes.

Results: TERT promoter and BRAF mutations were detected in 20/436 (4.6\%) and 257/434 thyroid nodules (59.2\%), respectively. At the end of the follow-up, 318/384 patients (82.8\%) had an excellent outcome, 48/384 (12.5\%) had indeterminate response or biochemical persistence, 18/384 (4.7\%) had a structural persistence or died from thyroid cancer. TERT promoter mutations correlated with older age $(P<0.0001)$, larger tumor size $(P=0.0002)$, oxyntic and aggressive PTC variants $(P=0.01)$, higher tumor stages $(P<0.0001)$, distant metastases $(<0.0001)$ and disease outcome $(P<0.0001)$. At multivariate analysis, TERT promoter mutation was not an independent predictor of disease outcome. TERT promoter mutation- (OR: 40.58; 95\% Cl: 3.06-539.04), and N1b lymph node metastases (OR: 40.16, 95\% Cl:

3.48-463.04) were independent predictors of distant metastases. BRAF mutation did not predict the outcome, and it correlated with a lower incidence of distant metastases $(P=0.0201)$.

Conclusions: TERT promoter mutation proved an independent predictor of distant metastases, giving clinicians the chance to identify many of the patients who warranted more aggressive initial treatment and closer follow-up.
\end{abstract}

\section{Introduction}

Follicular-derived thyroid cancer (FDTC) is the most common endocrine cancer (1), and it is being diagnosed more and more frequently, possibly due to the increasing use of neck ultrasound for thyroid diseases and other unrelated conditions (2). The prognosis for FDTC is usually good, the 10-year survival rate ranging between
85 and 93\% (1). There is a tendency to treat it using less invasive surgical procedures, and less radioiodine, with a weaker ${ }^{131}$ I activity (3). From 7 to $20 \%$ of FDTC recur or persist, however, especially if initial surgery is not radical $(3,4)$, and patients who require additional treatments and intensive follow-up may experience a higher morbidity 
and worse quality of life. The 5-year survival rate in cases of poorly-differentiated thyroid carcinoma (PDTC), and in patients with distant metastases is also significantly worse, at 72 and 30-50\%, respectively (5). Distant metastases are not very common, occurring in only $2-5 \%$ of cases, but they have a significant impact on patient outcome and quality of life (6). Unlike other cancers, in the case of FDTC, metastatic disease is amenable to treatment (providing the primary tumor has been completely removed) because it may respond to RAI therapy (3).

In the light of the above considerations, it is time to adjust surgical and radioiodine strategies to the individual risk of disease persistence or recurrence, distant metastases and death. This involves identifying the minority of patients with aggressive FDTC who warrant a more aggressive treatment and closer follow-up (2). Unfortunately, good prognostic indicators - available before surgery or already at the time of a patient's diagnosis - are still lacking.

Fine-needle aspiration (FNA) cytology has an essential role in the diagnosis of thyroid nodules, and the value of the molecular markers obtained thereby has been widely investigated, especially for indeterminate cytologies (3). The significance of $B R A F$ mutation as a prognostic molecular marker has also been investigated in numerous studies (7). According to some reports, BRAF mutations appear to be associated with large tumor size $(8,9)$, extension beyond the thyroid $(8,9)$, advanced stage at diagnosis $(8,10)$ and lymph node involvement $(9,11)$. How BRAF mutations correlate with outcome is a more complicated issue, however. A systematic meta-analysis found a significantly higher independent recurrence rate in BRAF V600E mutated than in BRAF wild-type tumors (11), but it is hard to say whether the BRAF mutation was an independent risk factor or whether said higher recurrence rate was due to aggressive clinicopathological features associated with the mutational status (3). BRAF mutation was found unassociated with any presence of distant metastases in most studies $(12,13,14)$. Sancisi et al. even found that distant metastases developed less frequently in BRAF-mutated than in wild-type tumors (15). Melo et al. confirmed as much from the molecular standpoint, finding BRAF mutation less often in distant metastases than in their paired primary tumors (16). Assessing BRAF mutation in isolation is therefore not enough for proper risk stratification (3).

Telomerase reactivation or re-expression is a hallmark of cancer and allows unlimited proliferation. Somatic mutations in the promoter region of telomerase reverse transcriptase (TERT) have been found in a large proportion of human tumors (16), including FDTC (17). In particular, the rates of TERT promoter mutation in thyroid specimens reportedly range from $7 \%(18,19)$ to $23 \%$ (20) in PTC, from $11.4 \%$ (17) to $32 \%$ (21) in FTC (with higher rates in aggressive cancers), from 29\% (19) to $43 \%$ (18) in PDTC and from $33 \%$ (19) to $51 \%$ in ATC (20). These mutations occur -124 and -146 base-pairs away from the TERT translation start site $[1,295,228 \mathrm{C}>\mathrm{T}$ (C228T) and 1,295,250 C>T (C250T)] (18). They increase TERT promoter activity, giving rise to new consensus sites for transcription factors. The literature has consistently demonstrated an association between TERT promoter mutation and older-aged patients, larger tumors, distant metastases and advanced stage at diagnosis $(18,19)$. On the other hand, TERT mutations do not seem to be associated with lymph node metastases $(18,19,22)$, and an association with extrathyroidal extension and vascular invasion has emerged in many, but not all reports (18, 19, 22). More importantly, TERT promoter mutations seem to independently predict patient mortality (19) and disease-free survival $(18,19,23)$, and this makes them seem promising as a way to identify tumors with more aggressive anatomopathological features and a worse prognosis. All the above-mentioned studies analyzed the TERT promoter in cancer tissue that became available after surgery, however. Its prognostic value in FNA from thyroid nodes prior to surgery remains unknown as yet, and the question is challenging, partly because TERT promoter mutations are often subclonal in thyroid cancer tissue (19). The aim of the present study was thus to elucidate the prognostic value of TERT promoter mutations in a large, single-center, consecutive series of cytologically malignant or suspect thyroid nodules.

\section{Patients and methods}

The study involved 436 samples found malignant or suspect on FNA cytology, obtained from thyroid nodules in 436 consecutive adult patients referred for surgical excision. Molecular analysis for somatic TERT promoter mutations was performed retrospectively in all patients. $B R A F$ analysis was performed as well for 434 patients. All studies were conducted in accordance with the guidelines of the Declaration of Helsinki. The present study was approved by our local ethical committee (Azienda Ospedaliera di Padova, approval code number: AOP1303), and all patients gave their written informed consent to the use of their thyroid cytology findings for research purposes. In the present series, the decision 
regarding the extent of initial surgery, considering total thyroidectomy and prophylactic neck compartment dissection, was based on patients' clinical status, and the surgeons' and patients' preferences: 434/436 patients (99.5\%) underwent total thyroidectomy, $1 / 436(0.2 \%)$ had a lobectomy and $1(0.2 \%)$ had no surgery due to a diagnosis of anaplastic thyroid carcinoma. All 436 cases collected were classified according to the SIAPEC 2014 consensus statement (24). ${ }^{131} \mathrm{I}$ ablation/therapy was performed in 387 patients (median dose: $100 \mathrm{mCi}$; range: 30-200 mCi). Histological diagnostics and staging were done according to the TNM classification, considering both the 7th (25), and the 8th (26) editions, and on the grounds of the first whole-body scan after ${ }^{131} \mathrm{I}$ remnant ablation. All patients with a negative whole-body scan outside the thyroid bed, negative thyroid ultrasound (US) and undetectable thyroglobulin (Tg) with negative thyroglobulin autoantibodies after therapy underwent rhTSH-stimulated Tg assessment 12 months after remnant ablation, according to standard procedures. Then patients were routinely followed up every 6 or 12 months. Additional Tg assays, FNA cytology or 18F-FDG PET were performed, depending on patients' clinical features or when persistent disease was suspected. Further ${ }^{131} \mathrm{I}$ and/ or surgical treatments, and/or external radiotherapy, and/ or tyrosine kinase inhibitor treatment were administered if further disease was confirmed. Patient outcome was classified as a 'biochemically incomplete or structurally incomplete response', 'indeterminate' or an 'excellent response', according to the American Thyroid Association (3) guidelines for patients undergoing ${ }^{131} \mathrm{I}$ administration, and according to the criteria proposed by Momesso et al. (27) for patients not receiving ${ }^{131}$ I treatment. The median patient follow-up was 59 months (range: 7-293 months); $39 / 423$ (9.2\%) patients with malignant disease were lost to follow-up.

\section{$B R A F$ and TERT mutation analysis}

DNA was isolated from FNA samples using the QIAamp DNA Micro kit (Qiagen) according to the manufacturer's protocol. BRAF (NM_004333.4) exon 15 and TERT proximal promoter (NM_198253.2) status were assessed by direct sequencing. The primers and PCR reaction protocol have been described elsewhere (28).

\section{Statistical analysis}

The Kolmogorov-Smirnov test was used to assess the normal distribution of each variable. Based on a 3.5- fold difference of adverse outcome in the two groups of patients (TERT promoter-mutated versus TERT promoter not mutated) and considering a mean frequency of TERT promoter mutation around 10\% in FDTC, as it comes from histological studies, a sample size of at least 341 patients was calculated ( $\alpha=0.05, \beta=0.2)$. The Mann-Whitney test and the Kruskal-Wallis test for nonparametric data were used to correlate age at diagnosis and cancer size with final outcome and mutational status, where appropriate. Categorical variables (gender, extrathyroidal extension, multifocality, vascular invasion, lymph node metastases, distant metastases, PTC histological variant, stage at diagnosis) were compared with outcome and mutational status using the chi-square test. Disease-free survival data were analyzed with the Kaplan-Meier method; multivariate analyses of factors affecting metastases and outcome were conducted using logistic regression. A $P$ value $<0.05$ was considered statistically significant.

\section{Results}

\section{Patients}

Of the 436 patients included in our study, 335 (76.8\%) were female and $101(23.2 \%)$ were male. The patients were a mean $47.8 \pm 13.6$ years old (median 47 years). There were $129 / 436$ patients (29.6\%) classified as TIR-4, and 307/436 (70.4\%) as TIR-5. Among 436 fine-needle aspirates, $423(97 \%)$ were classified as malignant on histopathological review. In detail, the histological classification of the malignant nodules was as follows: $282 / 423$ classical variant of papillary thyroid carcinoma (CV-PTC), 26/423 follicular variant of PTC (FV-PTC), 33/423 oxyphilic variant of PTC (VO-PTC), 70/423 aggressive variants of PTC, 7/423 follicular thyroid carcinoma (FTC), 2/423 PDTC, 2/423 anaplastic thyroid carcinoma (ATC) and 1 medullary thyroid carcinoma. All benign histologies (11/13 follicular adenomas and 2/13 hyperplastic nodules) were scored as TIR-4 at cytological examination. The size of the malignant nodules ranged from 5 to $60 \mathrm{~mm}$ (median $14 \mathrm{~mm}$ ), and 100/436 (22.9\%) were microcarcinomas (largest diameter $\leq 10 \mathrm{~mm}$ ). Prior to surgery, $14 / 85$ (16.5\%) of microcarcinomas were N1b. According to the 7th edition of the TNM, 313/423 patients (73.9\%) with malignant disease were classified in stage I, $44 / 423$ (10.5\%) in stage II, $47 / 423$ (11.1\%) in stage III and 19/423 (4.5\%) in stage IV at diagnosis. All patients were re-classified according to the 8th edition of the TNM as follows: $356 / 423(84.2 \%)$ in stage I, $60 / 423(14.2 \%)$ in 
stage II, $1 / 423(0.2 \%)$ in stage III and 6/423 (1.4\%) in stage IV. Eleven $(2.6 \%)$ of the 423 patients with a malignant histology had metastatic disease. Considering only the cases of FDTC, our series was classified according to the American Thyroid Association (ATA) guidelines of 2009 for the initial stratification of patients' cancer recurrence risk, as modified in 2015 (3): 135/422 (32\%) were low risk; 264/422 (65.6\%) were intermediate risk; and 23/422 (5.5\%) were high risk.

At the end of the follow-up, 318/384 patients (82.8\%) had an excellent outcome, 37/384 (9.6\%) had an indeterminate response, and 29/384 (7.6\%) had biochemically or structurally persistent disease or had died of their thyroid cancer (in 4/384 cases, 1\% of patients, two of them with ATC and two with PTC), while another two patients had died of other causes. For the purposes of our study, patient outcome was classified as: (i) excellent $318 / 384(82.8 \%)$ or (ii) indeterminate response and biochemically persistent disease $(48 / 384,12.5 \%)$ and (iii) structurally persistent disease and death due to thyroid cancer $(18 / 384,4.7 \%)$.

Primary tumor size $(P=0.001)$, extrathyroidal extension $(P=0.0007)$, vascular invasion $(P=0.0029)$, lymph node involvement $(P=0.0002)$ - with N1b carrying a higher risk than N1a $(P<0.0001)$, distant metastases $(P<0.0001)$, advanced stage at diagnosis (according to both the 7 th and the 8 th editions of the TNM, $P<0.0001$ ), and TERT promoter mutation $(P<0.0001)$ all correlated significantly with the risk of persistent/recurrent disease or disease-related death (Table 1). At multivariate analysis, only cancer size (OR 1.0459, 95\% CI 1.0006-1.0932), N1b lymph node metastases (OR 11.7323, 95\% CI 2.8167-48.8681) and distant metastases (OR 10.5559, 95\% CI 1.5767-70.6692) predicted persistent disease (i.e. a structurally incomplete response and disease-related death).

\section{BRAF mutation testing}

$B R A F$ mutations were detected in 257/434 thyroid nodules (59.2\%). The classic c.1799T $>$ A (p.V600E) mutation was found in 256 cases, and a c.1801A>G mutation (p.K601E) in one. Fifty-four (21\%) of the 257 patients carrying a BRAF mutation had a TIR-4 cytology, and 203/257 (79\%) had a malignant cytology; they all proved malignant on final pathology review. The results of the univariate analysis are summarized in Table 2. $B R A F$ mutation correlated with minimal extrathyroidal extension $(P=0.0174)$, multifocality $(P=0.0392)$, the PTC histological variant (the mutation being more prevalent in the classical and aggressive variants, $P<0.0001)$ and cancer size (the mutation being more frequent in small tumors with a median size of $13 \mathrm{~mm}$ in $B R A F$-mutated tumors as opposed to $15 \mathrm{~mm}$ in $B R A F$ wild-type tumors, $P=0.03)$. Sixty-three (63.3\%) of the 99 microcarcinomas carried a BRAF mutation. BRAF mutations correlated inversely with distant metastases: they were found in $3 / 11$ patients $(27.2 \%)$ with distant metastases, and in $254 / 410$ (61.9\%) of those without them $(P=0.021)$. BRAF mutation status also correlated with a lower incidence of second treatments $(P=0.0425)$, while it was not associated with disease outcome at the end of the follow-up.

\section{TERT promoter mutation testing}

TERT promoter mutations were detected in 20/436 (4.6\%) of cytologies. Nineteen of these 20 patients carried a C228T somatic TERT promoter mutation, and one had a C250T mutation. There was no overlap between the C228T and C250T mutations, the former being more prevalent than the latter. Eleven (55\%) of the 20 patients also carried a $B R A F$ mutation. Seven (35\%) of the 20 patients carrying a TERT promoter mutation had a TIR-4 cytology, while $13(65 \%)$ had a malignant cytology. The histological and clinical characteristics of the patients with TERT promoter mutations are given in Table 3. TERT promoter mutations were detected in 17/411 of the patients with PTC, in 1/7 of those with FTC, and in (1/2), of those with PDTC and ATC $(1 / 2)$. The results of the univariate analysis are summarized in Table 2. Following ATA Guidelines, patients with TERT promoter mutations were assigned to the following ATA risk categories: 4/20 (20\%) were ATA low risk; 12/20 (60\%) were intermediate risk and 4/20 (20\%) were high risk. TERT promoter mutations correlated with older age at diagnosis (median 68.5 years vs 46 years, $P<0.0001$ ), larger-sized primary tumors ( $25 \mathrm{~mm}$ vs $14 \mathrm{~mm}, P=0.0002$, with microcarcinomas all negative for TERT promoter mutations), the PTC histological variant (the mutation being more prevalent in the oxyntic and aggressive variants, $P=0.0079)$, advanced stage at diagnosis $(P<0.0001)$ and distant metastases $(P<0.0001)$. Extrathyroidal extension $(P=0.542)$, multifocality $(P=0.523)$, vascular invasion $(P=0.315)$ and lymph node metastases (0.954) did not correlate with TERT promoter mutation. When outcome was analyzed, the thyroid disease was structurally persistent or was the cause of death in 5/19 (26.3\%) TERT promoter-mutated cancers as opposed to $13(3.6 \%)$ of the 365 TERT promoter wild-type tumors $(P<0.0001)$. TERT promoter status was not an independent risk factor for disease persistence (structurally incomplete response and 
Table 1 Correlation between structurally incomplete response and thyroid cancer-related death versus biochemically incomplete response and indeterminate response versus excellent response and clinicopathological features of PTC and molecular status (univariate analysis).

\begin{tabular}{l}
\hline \\
n/total $n$ (\%) \\
Gender \\
M \\
F \\
Median years \\
Median tumor size mm \\
Extrathyroidal extension \\
Yes \\
No \\
Multifocality \\
Yes \\
No \\
Vascular invasion \\
Yes \\
No \\
PTC histological variants \\
CV \\
FV \\
OV \\
Other, aggressive \\
Lymph node metastases \\
Yes \\
No \\
Lymph node metastases \\
NO \\
N1a \\
Nes 1 b \\
No \\
Distant metastases \\
Yes \\
No \\
TNM stage 7th edition \\
I \\
II \\
III \\
IV \\
TNM stage 8th edition \\
I \\
II \\
III \\
TERT promoter mutation \\
Yos \\
No \\
No
\end{tabular}

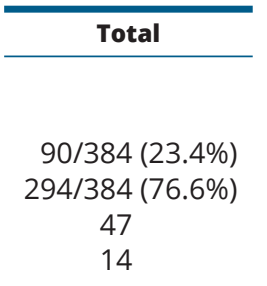

207/358 (57.8\%)

$151 / 358(42.2 \%)$

212/383 (55.4\%)

$171 / 383(44.6 \%)$

$130 / 203(64 \%)$

$73 / 203(36 \%)$

$254 / 373(68.1 \%)$

23/373 (6.2\%)

$30 / 373(8.0 \%)$

$66 / 373(17.7 \%)$

166/334 (49.7\%)

$168 / 334(50.3 \%)$

$168 / 334$ (50.3\%)

100/334 (29.9\%)

$66 / 334(19.8 \%)$

$11 / 384(2.9 \%)$

$373 / 384$ (97.1\%)

283/384 (73.7\%)

$39 / 384(10.2 \%)$

45/384 (11.7\%)

$17 / 384$ (4.4\%)

$325 / 384(84.6 \%)$

$53 / 384(13.8 \%)$

$1 / 384(0.3 \%)$

$5 / 384(1.3 \%)$

$19 / 384$

$365 / 384$

237/382 (62\%)

$145 / 382(38 \%)$

\begin{tabular}{c}
\hline SPD and TCD \\
\hline $18 / 384(4.7 \%)$ \\
$4 / 18(22.2 \%)$ \\
$14 / 18(77.8 \%)$ \\
49 \\
30
\end{tabular}

\section{$13 / 14(92.9 \%)$}

$1 / 14(7.1 \%)$

$12 / 17(70.6 \%)$

$5 / 17(29.4 \%)$

$11 / 11(100 \%)$

$0 / 11(0 \%)$

$8 / 15(53.3 \%)$

$1 / 15(6.7 \%)$

$1 / 15(6.7 \%)$

$5 / 15(33.3 \%)$

$14 / 16(87.5 \%)$

$2 / 16(12.5 \%)$

$2 / 16(12.5 \%)$

$2 / 16(12.5 \%)$

$12 / 16(75.0 \%)$

$7 / 18$ (38.9\%)

$11 / 18(61.1 \%)$

6/18 (33.3\%)

$7 / 18(38.9 \%)$

$0 / 18(0 \%)$

$5 / 18(27.8 \%)$

$8 / 18(44.4 \%)$

$7 / 18(38.9 \%)$

$0 / 18(0 \%)$

$3 / 18(16.7 \%)$

$5 / 18(27.8 \%)$

$13 / 18(72.2 \%)$

$9 / 18(50 \%)$

$9 / 18(50 \%)$

\begin{tabular}{c}
\hline IR and BPD \\
\hline $48 / 384(12.5 \%)$ \\
$10 / 48(20.8 \%)$ \\
$38 / 48(79.2 \%)$ \\
46 \\
15
\end{tabular}

$33 / 44(75 \%)$

$11 / 44(25 \%)$

$30 / 48$ (62.5\%)

$18 / 48$ (37.5\%)

$22 / 27$ (81.5\%)

$5 / 27(18.5 \%)$

$32 / 46(69.6 \%)$

$2 / 46(4.3 \%)$

$4 / 46(8.7 \%)$

$8 / 46(17.4 \%)$

28/41 (68.3\%)

$13 / 41(31.7 \%)$

13/41 (31.7\%)

15/41 (36.6\%)

$13 / 41(31.7 \%)$

$3 / 48(6.2 \%)$

45/48 (93.7\%)

$36 / 48(75 \%)$

$6 / 48(12.5 \%)$

$3 / 48(6.2 \%)$

$3 / 48(6.2 \%)$

$37 / 48(77.1 \%)$

$10 / 48(20.8 \%)$

$0 / 48(0 \%)$

$1 / 48(2.1 \%)$

$3 / 48(6.2 \%)$

45/48 (93.7\%)

$31 / 48(64.6 \%)$

$17 / 48$ (35.4\%) \begin{tabular}{c}
\hline Excellent response \\
\hline $318 / 384(82.8 \%)$ \\
$76 / 318(23.9 \%)$ \\
$242 / 318(76.1 \%)$ \\
47 \\
14
\end{tabular}

$161 / 300(53.7 \%)$

$139 / 300$ (46.3\%)

$170 / 318(53.5 \%)$

$148 / 318(46.5 \%)$

97/165 (58.8\%)

68/165 (41.2\%)

$214 / 312(68.6 \%)$

20/312 (6.4\%)

25/312 (8.0\%)

$53 / 312(17 \%)$

$124 / 277$ (44.8\%)

$153 / 277$ (55.2\%)

$153 / 277(55.2 \%)$

$83 / 277$ (30\%)

41/277 (14.8\%)

$1 / 318(0.3 \%)$

$317 / 318$ (99.7\%)

$241 / 318(75.8 \%)$

$26 / 318(8.2 \%)$

$42 / 318(13.2 \%)$

$9 / 318(2.8 \%)$

280/318 (88.1\%)

$36 / 318(11.3 \%)$

$1 / 318(0.3 \%)$

$1 / 318(0.3 \%)$

$11 / 318(3.5 \%)$

$307 / 318$ (96.5\%)

197/316 (62.3\%)

$119 / 316(37.7 \%)$

\begin{tabular}{c}
\hline P value \\
\hline 0.889
\end{tabular}

0.483

0.001

0.0007

0.218

0.0029

0.8073

0.0002

$<0.0001$

$<0.0001$

$<0.0001$

$<0.0001$

$<0.0001$

0.5347

CV-PTC, classical variant of papillary thyroid carcinoma; FV-PTC, follicular variant of papillary thyroid carcinoma; VO-PTC, oxyphilic variant of papillary thyroid carcinoma; IR, intermediate response; BPD, biochemically persistent disease; SPD, structurally persistent disease; TCD, death from thyroid cancer

disease-related death) at multivariate analysis, however. Interestingly, the frequency of TERT promoter mutation increased with worsening outcomes: mutations were present in $11 / 318$ patients (3.5\%) with an excellent response, in $3 / 48(6.3 \%)$ with a biochemically persistent disease or indeterminate response, in 3/14 (21.4\%) with structurally incomplete response, and in $4 / 11$ patients (36.4\%) with distant metastases and 2/4 (50\%) patients who died. In addition, 6 (31.6\%) of 19 patients with TERT promoter mutations required further treatment during their follow-up as opposed to $40 / 386(10.4 \%)$ of the wild-type cases $(P=0.045)$. 


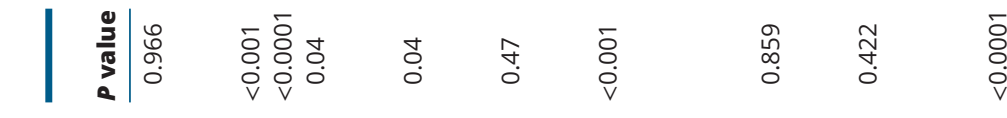

穿

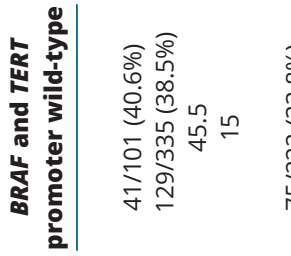

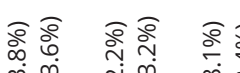

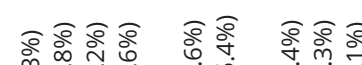

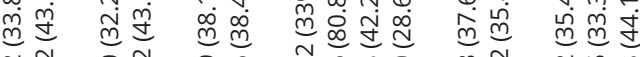

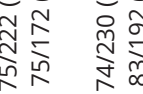

$\stackrel{m}{m}_{\substack{m \\ m}}^{\infty}$

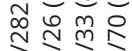

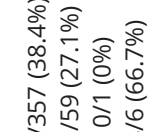

$\begin{array}{ll}\overline{8} & 0 \\ \vdots & 8 \\ 0 & 0\end{array}$

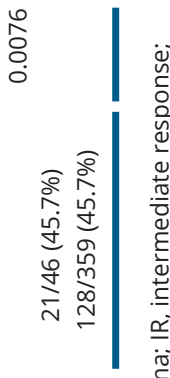

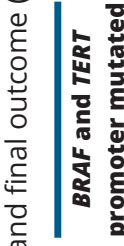

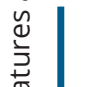

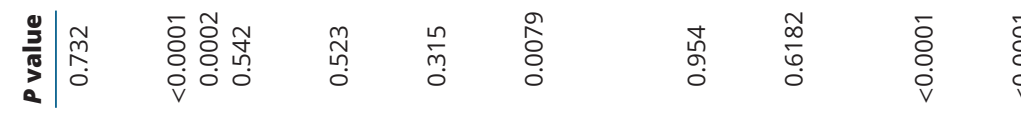

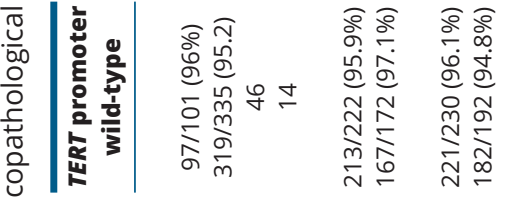

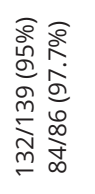

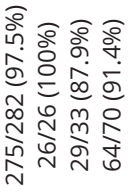

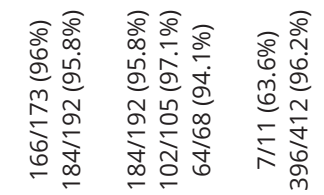

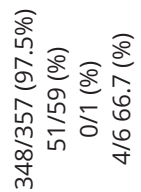

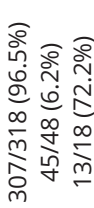

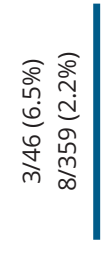

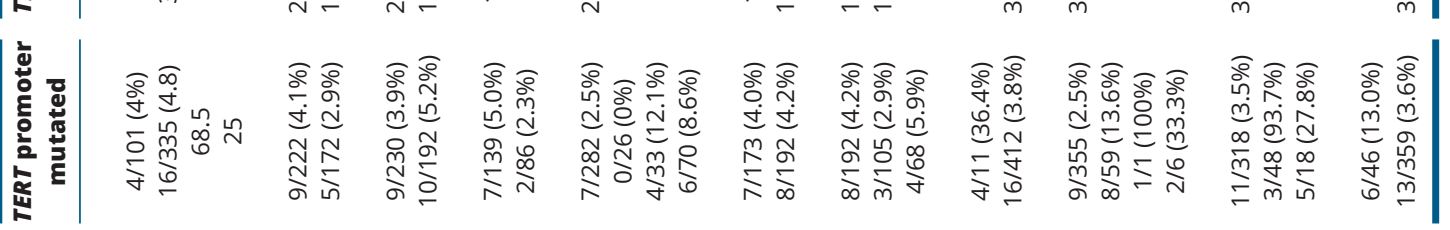

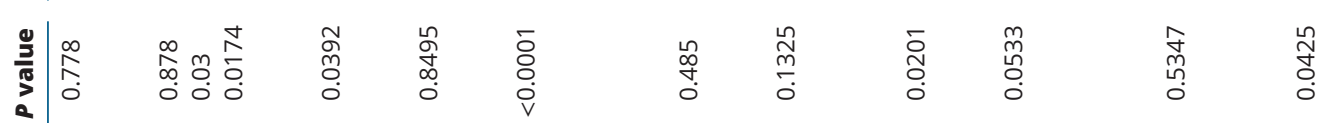

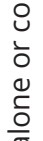

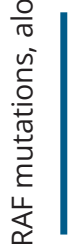

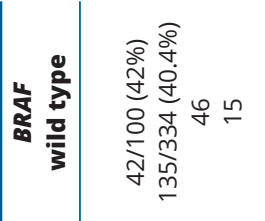

용

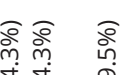

웅

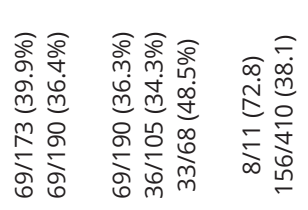

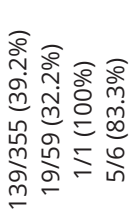

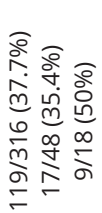

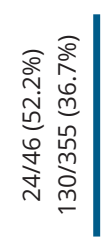

要

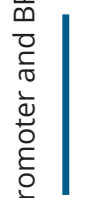

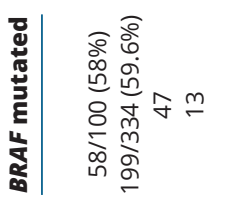

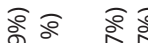

今े

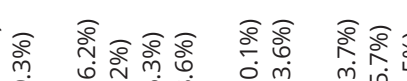

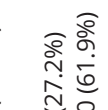

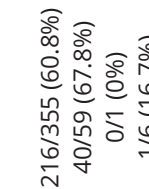

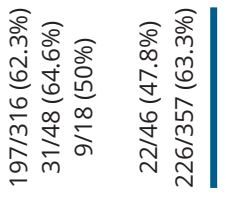

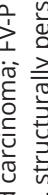

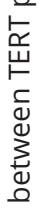

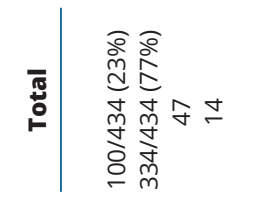

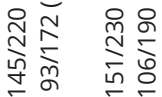

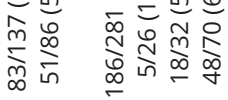

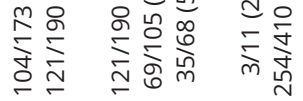

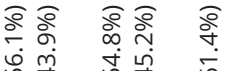

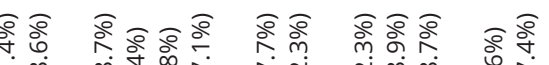

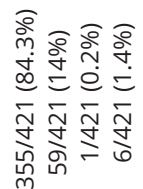

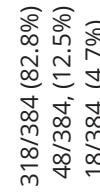

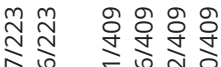

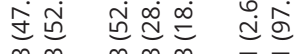

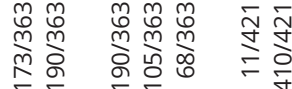

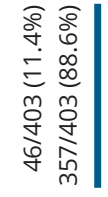

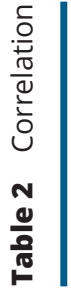

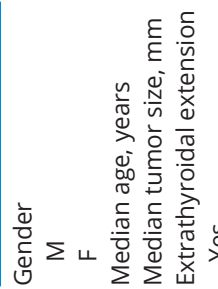

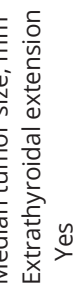

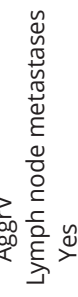

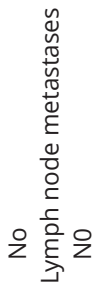

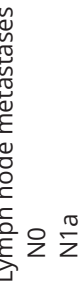

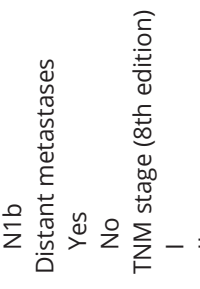

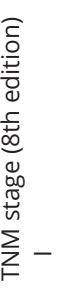

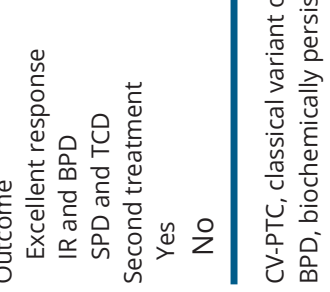




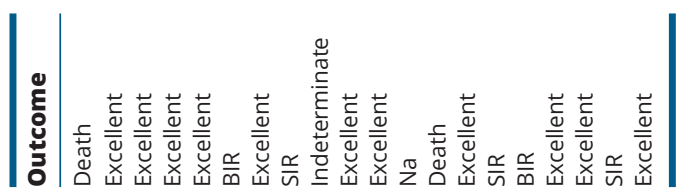

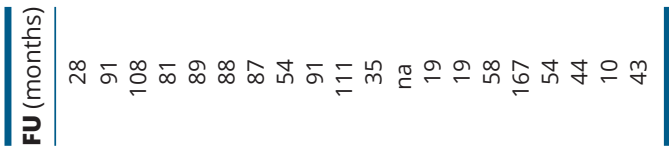

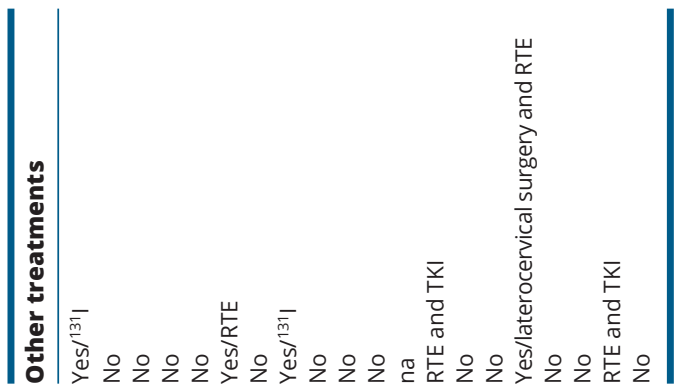

|亏

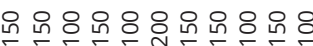

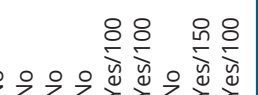

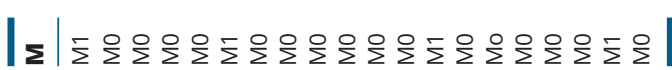

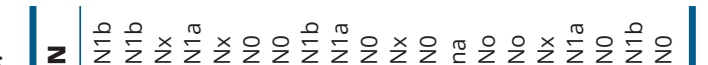

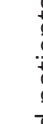

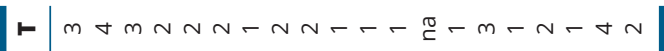

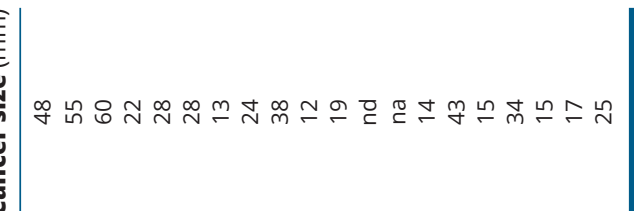

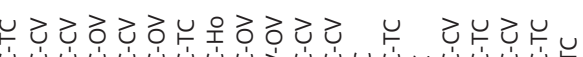

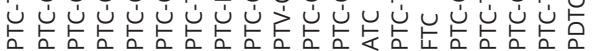

|

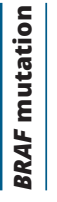

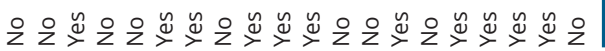

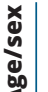

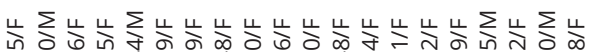
안

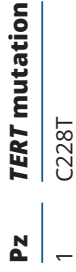

\section{Distant metastases}

When distant metastases were considered, older age at diagnosis $(P=0.0487)$, larger tumor size $(P=0.0015)$, extrathyroidal extension $(P=0.0120)$, lymph node involvement (both $\mathrm{N} 0$ versus $\mathrm{N} 1$, and $\mathrm{N} 0+\mathrm{N} 1$ a versus N1b; $P=0.0117$ and $P=0.0001$, respectively), and TERT promoter mutation $(P<0.0001)$ all correlated with M1 status (Table 4). On the other hand, BRAF-mutated tumors developed distant metastases less frequently than wild-type tumors $(P=0.0201)$. Intriguingly, only TERT promoter mutation (OR 40.58; 95\% CI 3.06-539.04) and N1b (versus N0 and N1a) (OR 40.16, 95\% CI 3.48-463.04) correlated independently with distant metastases at multivariate analysis. To be more precise as regards lymph node involvement, simply dichotomizing this variable as present or absent (N0 versus N1a and N1b) was unable to independently predict the presence of distant metastases at multivariate analysis.

\section{TERT promoter and BRAF testing}

The frequency of combined BRAF and TERT promoter mutations was also analyzed: $170 / 436$ patients (38.9\%) carried no mutations; 246/436 (56.4\%) had BRAF mutations; 9/436 (2.1\%) had TERT promoter mutations and $11 / 436$ (2.5\%) had both. The results are summarized in Table 2. The most interesting finding that emerged from this analysis regards the advanced age at diagnosis of patients carrying both mutations, who were significantly older (median 69 years) than patients with single mutations (46.5 and 60 years, respectively, for BRAF and TERT promoter mutations) or no mutations (45.5 years); $P<0.0001$.

\section{Discussion}

This is the first study, to our knowledge, on the frequency of TERT promoter mutations in a large series of suspect or frankly malignant thyroid cytologies. The first interesting finding that emerged concerns the overall frequency of TERT promoter mutations in this setting, which was only around $5 \%$. The overall rate of TERT mutations on histologically differentiated thyroid cancer specimens reportedly ranges from 7 to $23 \%(18,19,20,21)$, and the particularly low rate documented in our series may have several explanations. One important factor to consider is the subclonality of such mutations, which makes them more challenging to ascertain on cytological than on 
Table 4 Correlation between metastatic disease and clinicopathological and molecular features (univariate analysis).

\begin{tabular}{l}
\hline \\
\hline Gender \\
M \\
F \\
Median age, years \\
Median tumor size, mm \\
Extrathyroidal extension \\
Yes \\
No \\
Multifocality \\
Yes \\
No \\
Vascular invasion \\
Yes \\
No \\
PTC histological variants \\
CV \\
FV \\
OV \\
Other, aggressive \\
Lymph node metastases \\
NO \\
N1a and N1b \\
Lymph node metastases \\
NO \\
N1a \\
N1b \\
TERT promoter mutation \\
Yes \\
No \\
BRAF mutation \\
Yes \\
No \\
No \\
No
\end{tabular}

\begin{tabular}{c} 
Total \\
\hline $96 / 423(22.7 \%)$ \\
$327 / 423(77.3 \%)$ \\
47 \\
14 \\
$222 / 394(56.3 \%)$ \\
$172 / 394(43.7 \%)$ \\
$230 / 422(54.5 \%)$ \\
$192 / 422(45.5 \%)$ \\
$139 / 225(61.8 \%)$ \\
$86 / 225(38.2 \%)$ \\
$282 / 411(6.6 \%)$ \\
$26 / 411(6.3 \%)$ \\
$33 / 411(8.0 \%)$ \\
$70 / 411(17.0 \%)$ \\
$192 / 365(52.6 \%)$ \\
$173 / 365(47.4 \%)$ \\
$192 / 365(52.6 \%)$ \\
$105 / 365(28.8 \%)$ \\
$68 / 365(52.6 \%)$ \\
$20 / 423(4.7 \%)$ \\
$403 / 423(95.3 \%)$ \\
$257 / 421(61 \%)$ \\
$164 / 421(39 \%)$ \\
\hline \\
\\
\\
\end{tabular}

\begin{tabular}{c} 
M1 \\
\hline $2 / 96(2.1 \%)$ \\
$9 / 327(2.8 \%)$ \\
54 \\
28 \\
$8 / 222(3.6 \%)$ \\
$0 / 172(0 \%)$ \\
$7 / 230(3 \%)$ \\
$3 / 192(1.6 \%)$ \\
$3 / 139(2.2 \%)$ \\
$1 / 86(1.2 \%)$ \\
$5 / 282(1.8 \%)$ \\
$0 / 26(0 \%)$ \\
$1 / 33(3 \%)$ \\
$3 / 70(4.3 \%)$ \\
$1 / 192(0.5 \%)$ \\
$8 / 173(4.6 \%)$ \\
$1 / 192(0.5 \%)$ \\
$1 / 105(1 \%)$ \\
$7 / 68(10.3 \%)$ \\
$4 / 20(20 \%)$ \\
$7 / 403(1.7 \%)$ \\
$3 / 257(1.2 \%)$ \\
$8 / 164(4.9 \%)$ \\
\hline \\
\\
\\
\\
\end{tabular}

\begin{tabular}{|c|c|}
\hline Mo & $P$ value \\
\hline & $94 / 96$ (97.9\%) \\
\hline \multicolumn{2}{|l|}{$318 / 327(97.2 \%)$} \\
\hline 46 & 0.0487 \\
\hline \multirow[t]{2}{*}{14} & 0.0015 \\
\hline & 0.0120 \\
\hline \multicolumn{2}{|l|}{$214 / 222$ (96.4\%) } \\
\hline \multicolumn{2}{|l|}{$172 / 172(100 \%)$} \\
\hline & 0.3198 \\
\hline \multicolumn{2}{|l|}{$223 / 230(97 \%)$} \\
\hline \multicolumn{2}{|l|}{$189 / 192$ (98.4\%) } \\
\hline & 0.5838 \\
\hline \multicolumn{2}{|l|}{$136 / 139(97.8 \%)$} \\
\hline \multicolumn{2}{|l|}{ 85/86 (98.8\%) } \\
\hline & 0.502 \\
\hline \multicolumn{2}{|l|}{$277 / 282$ (98.2\%) } \\
\hline \multicolumn{2}{|l|}{$26 / 26(100 \%)$} \\
\hline \multicolumn{2}{|l|}{$32 / 33(97 \%)$} \\
\hline \multicolumn{2}{|l|}{$67 / 70(95.7 \%)$} \\
\hline & 0.0117 \\
\hline \multicolumn{2}{|l|}{$191 / 192$ (99.5\%) } \\
\hline \multicolumn{2}{|l|}{$165 / 173$ (95.4\%) } \\
\hline & 0.0001 \\
\hline \multicolumn{2}{|l|}{$191 / 192$ (99.5\%) } \\
\hline \multicolumn{2}{|l|}{$104 / 105(99 \%)$} \\
\hline \multicolumn{2}{|l|}{$61 / 68(89.7 \%)$} \\
\hline & $<0.0001$ \\
\hline \multicolumn{2}{|l|}{$16 / 20(80 \%)$} \\
\hline \multicolumn{2}{|l|}{$396 / 403$ (98.3\%) } \\
\hline & 0.0201 \\
\hline \multicolumn{2}{|l|}{$254 / 257(98.8 \%)$} \\
\hline $156 / 164$ (95.1\%) & \\
\hline
\end{tabular}

CV-PTC, classical variant of papillary thyroid carcinoma; FV-PTC, follicular variant of papillary thyroid carcinoma; VO-PTC, oxyphilic variant of papillary thyroid carcinoma.

histological specimens because genotyping on aspirates may not be representative of whole lesions. Another possible reason lies in the characteristics of our cytological series (obtained prior to surgery), which included a considerable proportion of microcarcinomas (22.9\%, all negative for TERT promoter mutation) and low-risk cancers (32\%), with a consequently low proportion of metastatic diseases. Taking all these concerns into account makes investigating the prognostic value of TERT promoter mutation even more intriguing. TERT promoter mutations were also found to correlate with older age, larger tumors, distant metastases, advanced tumor stage, persistent disease during the follow-up and a higher frequency of second treatments (Table 2). The close relationship between TERT promoter mutations and the presence of distant metastases is particularly noteworthy from a clinical point of view, as this molecular marker emerged as an independent predictor of distant metastases at multivariate analysis. The association between TERT promoter mutation in surgical specimens and metastatic thyroid disease has already been extensively described (6), but our study is the first to demonstrate that this applies when TERT promoter mutation is analyzed before any surgery as well. In such a presurgical setting, TERT promoter mutation correlated with higher rates of second treatments, and with persistent disease at the end of the follow-up, confirming the data obtained after surgery in the literature (19). Disease persistence also correlated with extrathyroidal extension, vascular invasion, lymph node metastases, distant metastases and advanced stage, but none of these variables (except for TERT promoter mutations and N1b) can be known prior to surgery. Hence the considerable potential of TERT promoter analysis as a prognostic molecular marker. The correlation with outcome was lost at multivariate analysis, however, when only cancer size, laterocervical lymph node involvement 
and distant metastases, but not TERT promoter mutation, were independent predictors of persistent disease. It is also worth noting that the rate of TERT promoter mutations rose with tumor aggressiveness (and dropped in patients with a good prognosis). Together with the association with older age and larger tumor size, this would suggest that TERT promoter mutation is a later genetic event in tumor carcinogenesis, giving tumors a more aggressive potential.

In short, the most important added value emerging from our study is that, like N1b status, TERT promoter mutations found at cytology on malignant and suspect thyroid nodules independently predict distant metastases. The presence of lateral neck compartment lymph node metastases is generally known before surgery and orients the choice of initial surgical approach: according to recent recommendations (recommendation 35 in the 2015 ATA Guidelines (3)), a more conservative surgical strategy is feasible in patients with clinical NO disease. TERT promoter mutation testing could be particularly useful in patients without any clinically evident lymph node involvement and with primary tumors less than $4 \mathrm{~cm}$ in size: given its association with distant metastases, a mutated TERT would promptly make such patients candidates for total thyroidectomy followed by ${ }^{131} \mathrm{I}$ administration.

In these times of personalized surgical management and treatment, knowing of a factor that predicts metastases already at the time of a thyroid malignancy's initial diagnosis would be particularly useful for identifying the small subset of aggressive thyroid cancers that warrant more extensive surgery, higher doses of ${ }^{131} \mathrm{I}$ and a closer follow-up.

A limitation of the present study concerns its retrospective nature, and the low frequency of TERT promoter mutations (5\%) in our consecutive series could limit its usefulness in clinical practice. Further data, also from high-risk and preferably prospective series, may help to clarify the cost-effectiveness of TERT mutation testing as a presurgical marker of distant metastases. On the other hand, the subclonality of TERT promoter mutations could result in a low frequency of this finding even in high-risk series. TERT promoter mutations were found more frequently in aggressive and oxyntic (or oncocytic) variants of PTC than in other variants, and we can offer no definitive explanation for this. The oncocytic features of thyroid cells stem from the accumulation of altered mitochondria in the cytoplasm, but the pathogenesis of oncocytic thyroid tumors is yet to be fully elucidated (29). There is plenty of evidence to suggest that oncocytic tumors follow a different genetic pathway from their nononcocytic counterparts $(30,31,32)$. The clinical behavior of this subtype is still controversial, however: it resembled that of typical PTC in many series (29), while some authors reported a more aggressive behavior, with higher rates of cancer recurrence and mortality $(33,34)$.

$B R A F$ mutations per se do not seem to be equally useful as a prognostic factor before surgery. In our consecutive series, they did not correlate with persistent disease, while they correlated inversely with distant metastases (Table 4) and the need for second treatments. This picture is in line with the results of other studies $(15,16,22)$. It may be that $B R A F$ mutations are early events in thyroid carcinogenesis. In fact, they were found in $63.3 \%$ of the microcarcinomas in our series (roughly the same proportion as the overall frequency of $B R A F$ mutations), and they did not give cancer cells a greater metastatic potential. BRAF mutations could only predict the presence of minimal extrathyroidal extension and multifocality. In $55 \%$ of the nodules with TERT promoter mutations, there were BRAF mutations too, confirming the association reported in the literature. As for the features of cancers involving both mutations, the combination of a BRAF mutation with a TERT promoter mutation did not make the cancer more aggressive than a TERT promoter mutation alone. Here again, our findings are in line with those coming from other series (19), and from a recent meta-analysis (6). These results should be considered with caution, however, given the small number of patients carrying both mutations. It is worth noting the association between older age and the presence of both mutations. Previous studies have also shown that the cancer-related mutational burden in solid tissue (be it malignant or benign) $(35,36)$ increases with age and is one of the hallmarks of senescence.

In conclusion, TERT promoter mutations identified on FNA cytology prior to surgery were found to correlate with aggressive phenotypes, although this mutation was not an independent predictor of disease outcome. Even in the cytological setting, TERT promoter mutation analysis was able to identify $36.4 \%$ of the patients with distant metastases and was thus an independent predictor of M1 status. It could therefore be used as a marker for risk stratification purposes and to guide a patient's surgical and radioiodine treatment. A possible weakness of such an approach lies in the relatively low frequency of TERT promoter mutations, which was only around $5 \%$ in our series. Obtaining further data from higher-risk series may help to clarify its cost-effectiveness as a presurgical marker of distant metastases. 


\section{Declaration of interest}

The authors declare that there is no conflict of interest that could be perceived as prejudicing the impartiality of this study.

\section{Funding}

This research did not receive any specific grant from any funding agency in the public, commercial or not-for-profit sector.

\section{Acknowledgement}

The authors thank Frances Coburn for text editing.

\section{References}

1 Hundahl SA, Fleming ID, Fremgen AM \& Menck HR. A National Cancer Data Base report on 53,856 cases of thyroid carcinoma treated in the U.S., 1985-1995. Cancer 199883 2638-2648. (https:// doi.org/10.1002/(SICI)1097-0142(19981215)83:12<2638::AIDCNCR31>3.0.CO;2-1)

2 Pacini F, Basolo F, Bellantone R, Boni G, Cannizzaro MA, De M, Durante C, Elisei R, Fadda G, Frasoldati A et al. Italian consensus on diagnosis and treatment of differentiated thyroid cancer: joint statements of six Italian societies. Journal of Endocrinological Investigation 201841 849-876. (https://doi.org/10.1007/s40618-0180884-2)

3 Haugen BR, Alexander EK, Bible KC, Doherty GM, Mandel SJ, Nikiforov YE, Pacini F, Randolph GW, Sawka AM, Schlumberger M et al. 2015 American Thyroid Association Management Guidelines for adult patients with thyroid nodules and differentiated thyroid cancer: the American Thyroid Association Guidelines task force on thyroid nodules and differentiated thyroid cancer. Thyroid 201626 1-133. (https://doi.org/10.1089/thy.2015.0020)

4 Grogan RH, Kaplan SP, Cao H, Weiss RE, Degroot LJ, Simon CA, Embia OMA, Angelos P, Kaplan EL \& Schechter RB. A study of recurrence and death from papillary thyroid cancer with 27 years of median follow-up. Surgery 2013154 1436-1446; discussion 1446-1447. (https://doi.org/10.1016/j.surg.2013.07.008)

5 Sampson E, Brierley JD, Le LW, Rotstein L \& Tsang RW. Clinical management and outcome of papillary and follicular (differentiated) thyroid cancer presenting with distant metastasis at diagnosis. Cancer 2007110 1451-1456. (https://doi.org/10.1002/cncr.22956)

6 Vuong HG, Altibi AM, Duong UN, Ngo HT, Pham TQ, Tran HM, Oishi N, Mochizuki K, Nakazawa T, Hassell L et al. Role of molecular markers to predict distant metastasis in papillary thyroid carcinoma: promising value of tert promoter mutations and insignificant role of BRAF mutations-a meta-analysis. Tumour Biology 201739 1010428317713913. (https://doi.org/10.1177/1010428317713913)

7 Tavares C, Melo M, Cameselle-Teijeiro JM, Soares P \& SobrinhoSimões M. ENDOCRINE TUMOURS: Genetic predictors of thyroid cancer outcome. European Journal of Endocrinology $2016 \mathbf{1 7 4}$ R117-R126. (https://doi.org/10.1530/EJE-15-0605)

8 Galuppini F, Pennelli G, Vianello F, Censi S, Zambonin L, Watutantrige-Fernando S, Manso J, Nacamulli D, Lora O, Pelizzo MR et al. BRAF analysis before surgery for papillary thyroid carcinoma: correlation with clinicopathological features and prognosis in a single-institution prospective experience. Clinical Chemistry and Laboratory Medicine 201654 1531-1539 . (https://doi.org/10.1515/ cclm-2015-0218)

9 Li C, Lee KC, Schneider EB \& Zeiger MA. BRAF V600E mutation and its association with clinicopathological features of papillary thyroid cancer: a meta-analysis. Journal of Clinical Endocrinology and Metabolism 201297 4559-4570. (https://doi.org/10.1210/ jc.2012-2104)

10 Tufano RP, Teixeira GV, Bishop J, Carson KA \& Xing M. BRAF mutation in papillary thyroid cancer and its value in tailoring initial treatment: a systematic review and meta-analysis. Medicine 201291 274-286. (https://doi.org/10.1097/MD.0b013e31826a9c71)

11 Xing M. Prognostic utility of BRAF mutation in papillary thyroid cancer. Molecular and Cellular Endocrinology 2010321 86-93. (https:// doi.org/10.1016/j.mce.2009.10.012)

12 Xing M, Liu R, Liu X, Murugan AK, Zhu G, Zeiger MA, Pai S \& Bishop J. BRAF V600E and tert promoter mutations cooperatively identify the most aggressive papillary thyroid cancer with highest recurrence. Journal of Clinical Oncology 201432 2718-2726. (https:// doi.org/10.1200/JCO.2014.55.5094)

13 Liu C, Chen T \& Liu Z. Associations between BRAFV600E and prognostic factors and poor outcomes in papillary thyroid carcinoma: a meta-analysis. World Journal of Surgical Oncology 2016 14 241. (https://doi.org/10.1186/s12957-016-0979-1)

14 Zhang Q, zheng LS, Zhang Q, xing GY, jie CQ \& yao ZQ. Metaanalyses of association between BRAF(600E) mutation and clinicopathological features of papillary thyroid carcinoma. Cellular Physiology and Biochemistry 201638 763-776. (https://doi. org/10.1159/000443032)

15 Sancisi V, Nicoli D, Ragazzi M, Piana S \& Ciarrocchi A. BRAFV600E mutation does not mean distant metastasis in thyroid papillary carcinomas. Journal of Clinical Endocrinology and Metabolism 201297 E1745-E1749. (https://doi.org/10.1210/jc.2012-1526)

16 Melo M, Gaspar da Rocha A, Batista R, Vinagre J, Martins MJ, Costa G, Ribeiro C, Carrilho F, Leite V, Lobo C et al. TERT, BRAF, and NRAS in primary thyroid cancer and metastatic disease. Journal of Clinical Endocrinology and Metabolism 2017102 1898-1907. (https:// doi.org/10.1210/jc.2016-2785)

17 Liu X, Bishop J, Shan Y, Pai S, Liu D, Murugan AK, Sun H, El-Naggar AK \& Xing M. Highly prevalent tert promoter mutations in aggressive thyroid cancers. Endocrine-Related Cancer 201320 603-610. (https://doi.org/10.1530/ERC-13-0210)

18 Liu R \& Xing M. Tert promoter mutations in thyroid cancer Endocrine-Related Cancer 201623 R143-R155. (https://doi. org/10.1530/ERC-15-0533)

19 Melo M, da Rocha AG, Vinagre J, Batista R, Peixoto J, Tavares C, Celestino R, Almeida A, Salgado C, Eloy C et al. Tert promoter mutations are a major indicator of poor outcome in differentiated thyroid carcinomas. Journal of Clinical Endocrinology and Metabolism 201499 E754-E765. (https://doi.org/10.1210/jc.2013-3734)

20 Landa I, Ganly I, Chan TA, Mitsutake N, Matsuse M, Ibrahimpasic T, Ghossein RA \& Fagin JA. Frequent somatic TERT promoter mutations in thyroid cancer: higher prevalence in advanced forms of the disease. Journal of Clinical Endocrinology and Metabolism 201398 E1562-E1566. (https://doi.org/10.1210/jc.2013-2383)

21 Liu X, Qu S, Liu R, Sheng C, Shi X, Zhu G, Murugan AK, Guan H, $\mathrm{Yu} \mathrm{H}$, Wang $\mathrm{Y}$ et al. TERT promoter mutations and their association with BRAF V600E mutation and aggressive clinicopathological characteristics of thyroid cancer. Journal of Clinical Endocrinology and Metabolism 201499 E1130-E1136. (https://doi.org/10.1210/ jc.2013-4048)

22 Gandolfi G, Ragazzi M, Frasoldati A, Piana S, Ciarrocchi A \& Sancisi V. TERT promoter mutations are associated with distant metastases in papillary thyroid carcinoma. European Journal of Endocrinology 2015172 403-413. (https://doi. org/10.1530/EJE-14-0837)

23 Kim TH, Ki CS, Kim HS, Kim K, Choe JH, Kim JH, Kim JS, Oh YL, Hahn SY, Shin JH et al. Refining dynamic risk stratification and prognostic groups for differentiated thyroid cancer with tert promoter mutations. Journal of Clinical Endocrinology and Metabolism 2017102 1757-1764. (https://doi.org/10.1210/jc.2016-3434) 
24 Nardi F, Basolo F, Crescenzi A, Fadda G, Frasoldati A, Orlandi F, Palombini L, Papini E, Zini M, Pontecorvi A et al. Italian consensus for the classification and reporting of thyroid cytology. Journal of Endocrinological Investigation 201437 593-599. (https://doi. org/10.1007/s40618-014-0062-0)

25 Wittekind C \& Sobin LGM. TNM: classification of malignant tumours. In TNM Classification of Malignant Tumours, 7th ed. 2009. Wiley-Blackwell: Oxford, UK

26 Tuttle RM, Haugen B \& Perrier ND. Updated American Joint Committee on cancer/tumor-node-metastasis staging system for differentiated and anaplastic thyroid cancer (eighth edition): what changed and why? Thyroid 201727 751-756. (https://doi. org/10.1089/thy.2017.0102)

27 Momesso DP, Vaisman F, Yang SP, Bulzico DA, Corbo R, Vaisman M \& Tuttle RM. Dynamic risk stratification in patients with differentiated thyroid cancer treated without radioactive iodine. Journal of Clinical Endocrinology and Metabolism 2016101 2692-2700. (https://doi. org/10.1210/jc.2015-4290)

28 Barollo S, Pezzani R, Cristiani A, Redaelli M, Zambonin L, Rubin B, Bertazza L, Zane M, Mucignat-Caretta C, Bulfone A et al. Prevalence, tumorigenic role, and biochemical implications of rare BRAF alterations. Thyroid 201424 809-819. (https://doi.org/10.1089/ thy.2013.0403)

29 Asa SL. My approach to oncocytic tumours of the thyroid. Journal of Clinical Pathology 200457 225-232. (https://doi.org/10.1136/ JCP.2003.008474)

30 Tallini G, Hsueh A, Liu S, Garcia-Rostan G, Speicher MR \& Ward DC. Frequent chromosomal DNA unbalance in thyroid oncocytic (Hürthle cell) neoplasms detected by comparative genomic hybridization. Laboratory Investigation 199979 547-555.

31 de Vries MM, Celestino R, Castro P, Eloy C, Máximo V, van der Wal JE, Plukker JTM, Links TP, Hofstra RMW, Sobrinho-Simões M et al. RET/PTC rearrangement is prevalent in follicular Hürthle cell carcinomas. Histopathology 201261 833-843. (https://doi. org/10.1111/j.1365-2559.2012.04276.x)

32 Jacques C, Guillotin D, Fontaine JF, Franc B, Mirebeau-Prunier D, Fleury A, Malthiery Y \& Savagner F. DNA microarray and miRNA analyses reinforce the classification of follicular thyroid tumors. Journal of Clinical Endocrinology and Metabolism 201398 E981-E989. (https://doi.org/10.1210/jc.2012-4006)

33 Herrera MF, Hay ID, Wu PS, Goellner JR, Ryan JJ, Ebersold JR, Bergstralh EJ \& Grant CS. Hürthle cell (oxyphilic) papillary thyroid carcinoma: a variant with more aggressive biologic behavior. World Journal of Surgery 199216 669-674; discussion 774-775. (https://doi. org/10.1007/BF02067351)

34 Hong JH, Yi HS, Yi S, Kim HW, Lee J \& Kim KS. Implications of oncocytic change in papillary thyroid cancer. Clinical Endocrinology 201685 797-804. (https://doi.org/10.1111/cen.13115)

35 Milholland B, Auton A, Suh Y \& Vijg J. Age-related somatic mutations in the cancer genome. Oncotarget $2015624627-24635$. (https://doi.org/10.18632/oncotarget.5685)

36 Risques RA \& Kennedy SR. Aging and the rise of somatic cancer-associated mutations in normal tissues. PLoS Genetics 201814 e1007108. (https://doi.org/10.1371/journal. pgen.1007108)

Received 31 January 2019

Revised version received 19 April 2019

Accepted 29 April 2019 